\title{
Microarrays identify matrix metalloproteinases (MMP) as key genes whose expression is up regulated in human adipocytes by macrophage-conditioned media
}

\author{
A. O'Hara ${ }^{1}$, F.-L. Lim ${ }^{2}$, D. Mazzatti ${ }^{2}$ and P. Trayhurn ${ }^{1}$ \\ ${ }^{1}$ Obesity Biology Research Unit, School of Clinical Sciences, University of Liverpool, Liverpool L69 3GA, UK and \\ ${ }^{2}$ Unilever R\&D Discover, Colworth Park, Bedfordshire MK44 1LQ, UK
}

A major development in obesity research is the recognition that the condition is characterized by chronic mild inflammation ${ }^{(1,2)}$. Within adipose tissue this process involves the infiltration of macrophages as well as a direct inflammatory response of adipocytes. In the present study DNA microarrays and conditioned media have been employed to examine the effects of macrophages on global gene expression in human adipocytes, and particularly of genes associated with inflammation.

Human SGBS cells (adipocytes), differentiated in culture, were treated with macrophage (U937 cells)-conditioned medium (MC medium) for 4 or $24 \mathrm{~h}$. Control cells were treated with unconditioned medium (UC medium). Agilent arrays comprising 44000 probes were used to analyse gene expression. Real-time PCR with Taqman probes was used both to validate the arrays and to quantify specific mRNA. An ELISA was used to measure the release of MMP1.

Microarray analysis identified $>5000$ genes that were either up or down regulated in adipocytes by incubation in the presence of MC medium. Of these genes, 1088 were identified as being differentially expressed in response to the MC medium at both 4 and $24 \mathrm{~h}$; most (754) genes were up regulated. This substantial group of genes particularly included those associated with inflammation and macrophage infiltration, such as IL-6, (365-fold increase at $24 \mathrm{~h} ; P<0.01)$, IL-8 $(120$-fold increase at $24 \mathrm{~h} ; P<0.01)$ and monocyte chemoattractant protein-1 (20-fold increase at $24 \mathrm{~h} ; P<0.01)$. Further analysis identified a cluster of MMP genes that were highly up regulated at both time points; these genes included MMP1, 3, 9, 10, 12 and 19. At both 4 and $24 \mathrm{~h} \mathrm{MMP1}$ was the most highly up regulated gene (>2400-fold increase in mRNA at $24 \mathrm{~h} ; P<0.01)$. ELISA measurements indicated that substantial quantities of MMP1 were released from SGBS adipocytes incubated with the MC medium ( $>290 \mathrm{ng} / \mathrm{ml}$ over $24 \mathrm{~h}$ ), with no release in control adipocytes or adipocytes incubated with UC medium. Treatment of adipocytes with TNF $\alpha(100 \mathrm{ng} / \mathrm{ml})$ led to a substantial increase $(>100$-fold; $P<0.01)$ in MMP1 mRNA level in SGBS adipocytes, suggesting that this cytokine could contribute to the stimulation of MMP1 expression by macrophages.

In conclusion, MC medium induces a major inflammatory response in human adipocytes, with expression of MMP gene family members being markedly up regulated. The role of MMP in adipocytes is not fully understood; however, the strong induction of MMP1 and other MMP suggests that macrophages stimulate tissue remodelling within adipose tissue in obesity.

A. O'H. is in receipt of an Industrial CASE Studentship from the Biotechnology and Biological Sciences Research Council.

1. Trayhurn P \& Wood IS (2004) Br J Nutr 92, 347-355.

2. Trayhurn P, Wang IS \& Wood (2008) Br J Nutr 100, 227-235. 